\title{
DINÂMICA DO BANCO DE PLÂNTULAS DE PALMITO EM AMBIENTE NATURAL
}

\section{DYNAMICS OF THE PALM TREE SEEDLING BANK IN NATURAL ENVIRONMENT}

\author{
Emerson Luiz TONETTI ${ }^{1}$ \\ Raquel Rejane Bonato NEGRELLE ${ }^{2}$ :
}

\begin{abstract}
RESUMO
O trabalho foi desenvolvido na propriedade da BANESTADO S/A Reflorestadora, em Paranaguá, PR ( $25^{\circ} 35^{\prime} \mathrm{S}$; $\left.48^{\circ} 32^{\prime} \mathrm{O}\right)$, visando contribuir para o conhecimento da biologia de Euterpe edulis Mart. em florestas naturais e auxiliar futuros planos de manejo da espécie. Especificamente buscou-se estimar, para o período de um ano, o crescimento, a sobrevivência, a natalidade realizada e a transição dos estádios de desenvolvimento do conjunto de indivíduos sem estipe exposto (banco de plântulas). O padrão de sobrevivência encontrado assemelha-se àqueles já descritos na literatura, representado por alta mortalidade entre os estádios mais jovens. A mortalidade decorrente de danos físicos causados pela queda de galhos ou folhas foi expressiva. Registrou-se que o crescimento em altura, diâmetro e produção de folhas tende a elevar-se do estádio de plântula para jovem II. O mesmo pôde ser observado para o número de folhas mortas nesses estádios. O registro de entrada de novos indivíduos na população e os valores de sobrevivência e transição obtidos para os diferentes estádios estudados são indicativos da franca regeneração natural da espécie no local do estudo.

Palavras-chave: Floresta Attântica, extrativismo sustentável, produtos florestais não madeiráveis.
\end{abstract}

\begin{abstract}
The work was carried out at BANESTADO S/A Reflorestadora, in Paranaguá, Paraná $\left(25^{\circ} 35^{\prime}\right.$ S; $\left.48^{\circ} 32^{\prime} \mathrm{O}\right)$. Our intent was to contribute to the biology knowledge of Euterpe edulis Mart. in natural environment, to support future sustainable management plans. Specifically, we sought to estimate growth, survival, birth and transition rates of the juveniles (seedlings bank) for the period of one year. The survival pattern observed was similar to the ones already described in the literature, represented by high mortality rate among younger stages. The mortality caused by falling branches was very expressive. It was verified that growth in height, diameter and leaf production tend to increase at the older stages. The same holds true for the number of dead leaves. The birth, survival and transition rates obtained for the different stages under study indicate spontaneous natural regeneration of this species at the study site.
\end{abstract}

Key words:Atlantic Forest, sustainable harvest, non-wood forest products.

Biólogo, M.Sc. Botânica.

Bióloga, Doutora, Universidade Federal do Paraná, Professora, Departamento de Botânica, Caixa Postal 19031, CEP 81531-970, Curitiba, PR. E-mail: rbgrelle@cce.ufpr.br =- Autor para correspondência. 


\section{INTRODUÇÃO}

Entre as diversas espécies florestais com potencial extrativista, o palmiteiro ou palmito (Euterpe edulis Mart.) tem sido muito indicado para estudos de manejo sustentado [5, 14, 15]. Devido, entre outras qualidades, a sua ampla distribuição geográfica, por ocupar o estrato médio da floresta, ter grande produção de sementes, apresentar forte interação dentro da comunidade e pela fácil comercialização de seus produtos [14].

Essa espécie é considerada ecologicamente importante para a comunidade em função do elevado nível de interação com a fauna [14, 15, 20], servindo de alimento para muitas espécies. Sua importância econômica deve-se principalmente a sua utilização no preparo de conservas pelas indústrias alimentícias, tendo larga aceitação no mercado interno e externo. Representou, em décadas passadas, grande potencial para a indústria de conservas.

Com a exploração contínua e sem reposição, os estoques naturais diminuíram expressivamente [11]. Ainda hoje essa atividade contribui informalmente como uma das principais fontes de renda para muitos habitantes do litoral paranaense, sendo na maioria das vezes praticada por pequenas fábricas clandestinas. A exploração é feita, especialmente, pelo corte indiscriminado do estoque natural dos indivíduos existentes, sem a preocupação de promover a manutenção das populações da espécie [17].

Para garantir a exploração sustentável, a regeneração natural da espécie deve ser considerada [15]. Nesse contexto é de fundamental importância o conhecimento das condições de crescimento, durante os primeiros estádios, especialmente quando se tratar de uma espécie que, como o palmito, ocorre em formações heterogêneas [16].

O presente trabalho pretende contribuir para o conhecimento da biologia do palmito em florestas naturais, visando auxiliar futuros planos de manejo para a espécie. Especificamente, buscou-se estimar, para o período de um ano, o crescimento, a sobrevivência, a natalidade realizada e a transição dos estádios de desenvolvimento do conjunto de indivíduos sem estipe exposto, que representam o banco de plântulas da espécie.

\section{METODOLOGIA}

O trabalho foi desenvolvido em área da empresa BANESTADO S/A Reflorestadora (1.780 ha), em Paranaguá, PR ( $\left.25^{\circ} 35^{\prime} \mathrm{S} ; 48^{\circ} 32^{\prime} \mathrm{O}\right)$, situada na planície costeira do Estado, onde ocorre, naturalmente, a Floresta Ombrófila Densa de Terras Baixas [24]. A pesquisa restringiu-se a um dos locais cobertos por floresta nativa sem evidências visuais ou históricas de haver sido submetida a corte raso ou mesmo seletivo intenso. O solo dessa área foi identificado com um Podzol [22]. O clima dessa região é caracterizado como tropical, superúmido, sem estação seca e isento de geadas, onde o mês mais quente apresenta temperatura média acima de $22^{\circ} \mathrm{C}$ e o mês mais frio com média acima de $18^{\circ} \mathrm{C}[8]$.

Os palmitos sem estipe exposto foram amostrados em área de 0,1 ha, adaptando-se o método proposto por Gentry (1982) [6], que consistiu na delimitação de dez transectos distribuídos aleatóriamente dentro do quadrado de um hectare previamente demarcado. Cada transecto possuía $2 \mathrm{~m}$ de largura e $50 \mathrm{~m}$ de comprimento, todos paralelos entre si e separados pela distância mínima de $3 \mathrm{~m}$. A instalação dos transectos e a coleta dos dados foram realizadas durante a primeira quinzena de fevereiro de 1995 (primeiro censo) e 1996 (recenso). Nesses censos, para cada indivíduo foram obtidas as seguintes informações: a) altura medida desde o solo até o ponto de separação da "folha flecha" e a primeira folha aberta, com régua milimétrica; b) diâmetro da base - obtido pela média de duas medidas feitas com paquímetro (uma medida sendo perpendicular a outra) no nível do solo ou logo acima das raízes; e c) número de folhas saudáveis - obtido pela contagem do total de folhas completamente expandidas, com mais de $50 \%$ de aparência saudável, isto é, sem evidências de patogenias. No recenso, fez-se mensuração e marcação dos novos indivíduos que se estabeleceram depois do primeiro censo.

Os estádios de desenvolvimento adotados foram baseados na proposta de Reis (1987) [14], considerando-se: a) plântulas - indivíduos com até $10 \mathrm{~cm}$ de altura de inserção da folha flecha tendo, geralmente, apenas uma folha de forma flabeliforme e a grande maioria apresentando reservas endospermáticas; b) planta jovem I - indivíduos maiores que $10 \mathrm{~cm}$ de altura de inserção e menores que $30 \mathrm{~cm}$, com número variável de folhas (geralmente duas a quatro) sendo as inferiores flabeliformes e as superiores pinadas e que se apresentam independentes das reservas da semente; e c) planta jovem II - indivíduos entre $30 \mathrm{~cm}$ e $1 \mathrm{~m}$ de altura de inserção, sem o estipe exposto e com quatro a cinco folhas pinadas.

A taxa de sobrevivência do estádio $i\left(\sigma_{i}\right)$ e a taxa de transição do estádio $i$ para o próximo $\left(\gamma_{i}\right)$ foram calculadas a partir das seguintes fórmulas retiradas de Silva-Matos (1995) [18]: $\sigma_{i}=\operatorname{Si} / \mathrm{n} i$ e $\gamma_{i}=$ ri/S $i$ onde $S i=$ número de plantas que iniciaram no estádio $i$ e sobreviveram durante 0 ano; $\mathrm{n} i=$ número de plantas vivas que iniciaram o ano no estádio $i ; r i=$ número de plantas que deixaram o estádio $i$, passando para o próximo estádio, após um ano.

A natalidade realizada foi obtida pela contagem do número de novos indivíduos registrados no recenso. O crescimento foi analisado a partir das médias da variação anual em altura, diâmetro e produção de folhas e por gráficos de freqüência das classes de crescimento de cada uma dessas características, em cada um dos estádios. Foi possível também, como conseqüência do acompanhamento da produção de folhas, analisar a mortalidade de folhas dos indivíduos nos diferentes 
estádios.

\section{RESULTADOS}

\section{Sobrevivência, transição e natalidade}

No total foram registrados 226 indivíduos em 1995 e 304 em 1996, pertencentes ao banco de plântulas. Os representantes dos estádios de plântula e jovem I perfizeram $93,65 \%$ do total amostrado em 1995 e 94,63\% em 1996 (Tabela 1). A proporção de indivíduos nos diferentes estádios manteve-se a mesma para os anos de 1995 e 1996 $(\chi 2=2,02 ; \mathrm{gl}=2$ e $\mathrm{p}=0,36)$.

Com exceção do estádio jovem II que apresentou $100 \%$ de sobrevivência no período observado, os demais estádios apresentaram diferentes porcentagens de sobrevivência. Entre estes, destaca-se o estádio plântula por apresentar o menor índice de sobrevivência (Tabela 2). Para o total de indivíduos mortos nos três estádios iniciais avaliados, $20,6 \%$ das mortes foram reconhecidas como resultantes da queda de galhos ou folhas sobre os indivíduos. Não foi possível reconhecer a causa da morte dos outros indivíduos.

TABELA 1 - Descritores estruturais do banco de plântulas de Euterpe edulis, baseados nas coletas das unidades amostrais de $1 \mathrm{~m}^{2}$, Paranaguá, PR, 1995/96 ${ }^{1}$

\begin{tabular}{lccccrrrr}
\hline \multirow{2}{*}{ Estádio } & \multicolumn{2}{c}{$\begin{array}{c}\text { Número de } \\
\text { indivíduos }\end{array}$} & \multicolumn{2}{c}{$\begin{array}{c}\text { Densidade média } \\
\text { (plantas/subparcela) }\end{array}$} & \multicolumn{2}{c}{ Freqüência (\%) } & \multicolumn{2}{c}{ Índice de Morisita } \\
& \multicolumn{1}{c}{1995} & 1996 & 1995 & 1996 & 1995 & 1996 & 1995 & 1996 \\
\hline Plântula & 102 & 156 & $0,102(0,34)^{2}$ & $0,156(0,54)$ & 9,2 & 11,2 & 2,32 & 6,86 \\
Jovem I & 118 & 140 & $0,118(0,39)$ & $0,14(0,43)$ & 9,7 & 11,4 & 3,62 & 3,39 \\
Jovem II & 6 & 8 & $0,006(0,07)$ & $0,008(0,08)$ & 0,6 & 0,8 & $0,0^{*}$ & $0,0^{*}$ \\
Total & 226 & 304 & $0,226(0,55)$ & $0,304(0,75)$ & 18 & 21,5 & 2,59 & 4,0 \\
\hline
\end{tabular}

1 valores não significativos com $95 \%$ de confiança

2 números entre parênteses correspondem ao desvio padrão

TABELA 2 - Taxas de sobrevivência ( $\sigma i)$ e transição para o próximo estádio $(\gamma i)$ dos indivíduos do banco de plântulas de palmito, Paranaguá, PR, 1995/96

\begin{tabular}{|c|c|c|c|c|c|}
\hline Estádios & ni & $\mathrm{Si}$ & $\sigma i \times 100$ & ri & $\gamma \mathrm{i} \times 100$ \\
\hline Plântula & 102 & 73 & 71,5 & 29 & 39,7 \\
\hline Jovem I & 118 & 113 & 95,7 & 2 & 1,76 \\
\hline Total & 226 & 192 & 84,9 & 31 & 16,1 \\
\hline
\end{tabular}

ni - número de plantas vivas que iniciaram o ano no estádio i;

$\mathrm{Si}$ - número de plantas que iniciaram no estádio i e sobreviveram durante o ano;

ri - número de plantas que deixaram o estádio i, passando para o próximo estádio, após um ano.

A maior taxa de transição foi registrada para o estádio de plântula, onde quase $40 \%$ dos indivíduos passaram para o estádio jovem I (Tabela 2). A natalidade realizada para o período de doze meses foi de 112 indivíduos, sendo que todos esses novos indivíduos se enquadravam na descrição do estádio de plântula.

\section{Crescimento}

Registrou-se aumento crescente das médias do crescimento em altura e diâmetro do estádio de plântula para jovem II. Esse crescimento em altura e diâmetro tende a ser maior nos estádios de maior tamanho (jovem I e jovem II), mesmo quando se avaliam as medianas (Tabela 3). Esse resultado também foi corroborado pela análise das freqüências das classes de crescimento em cada um dos estádios. Os estádios de plântula e jovem I concentraram-se nas menores classes de crescimento, enquanto que os indivíduos do estádio jovem II encontravam-se nas maiores classes. Observou-se, também, que os indivíduos do estádio jovem I tinham maior freqüência das classes de crescimento intermediárias quando comparadas com as freqüências do estádio de plântula (Figuras 1 e 2). A maioria dos indivíduos, em todos os estádios do banco de plântulas, produziu uma folha nova durante o ano, alguns não produziram novas folhas e outros produziram até duas folhas (Figura 3). Esse padrão de produção de folhas leva à mesma interpretação feita quanto ao crescimento em altura e diâmetro, reforçando a existência da tendência de aumento do crescimento do estádio de plântula para jovem II.

Para a maioria dos indivíduos do estádio de plântula não foi detectada a ocorrência de folhas mortas. Nos outros dois estádios a maioria dos indivíduos apresentou pelo menos uma folha morta. Para o estádio jovem I, registrou-se sensível aumento do número de duas folhas mortas em relação ao estádio de plântula (Figura 3 e Tabela 4). A ocorrência de três ou quatro folhas mortas durante o ano esteve associada à queda de galhos ou folhas 
TABELA 3 - Estatísticas descritivas do crescimento em altura e diâmetro dos estádios do banco de plântulas de palmito, Paranaguá, PR, 1995/96

\begin{tabular}{|c|c|c|c|c|}
\hline Altura $(\mathrm{mm})$ & Plântula & Jovem I & Jovem II & Total \\
\hline Número & 32 & 111 & 6 & 149 \\
\hline Média & 14,93 & 24,93 & 82,33 & 25,1 \\
\hline Desvio Padrão & 12,15 & 23,48 & 33,82 & 25,19 \\
\hline Coeficiente de Variação (\%) & 81,38 & 94,18 & 41,00 & 100,35 \\
\hline Erro Padrão & 2,14 & 2,22 & 13,80 & 2,06 \\
\hline Valor Mínimo & 0 & 0 & 29,00 & 0 \\
\hline Mediana & 12,50 & 18,00 & 82,50 & 18,00 \\
\hline Valor Máximo & 48,00 & 112,00 & 133,00 & 133,00 \\
\hline \multicolumn{5}{|l|}{ Diâmetro $(\mathrm{mm})$} \\
\hline Número & 61 & 94 & 6 & 161 \\
\hline Média & 0,59 & 1,01 & 5,11 & 1,01 \\
\hline Desvio Padrão & 0,40 & 0,68 & 2,82 & 1,13 \\
\hline Coeficiente de Variação (\%) & 67,79 & 66,33 & 55,10 & 110,89 \\
\hline Erro Padrão & 0,05 & 0,07 & 1,15 & 0,09 \\
\hline Valor Mínimo & 0 & 0 & 1,45 & 0 \\
\hline Mediana & 0,52 & 0,87 & 4,58 & 0,70 \\
\hline Valor Máximo & 1,65 & 3,35 & 9,50 & 9,50 \\
\hline
\end{tabular}

TABELA 4 - Estatísticas descritivas do número de folhas novas e do número de folhas mortas dos estádios do banco de plântulas de palmito.

\begin{tabular}{lcccr}
\hline Produção de folhas & Plântula & Jovem I & Jovem II & Total \\
\hline Número & 73 & 113 & 6 & 192 \\
Valor Mínimo & 0 & 0 & 1 & 0 \\
Moda & 1 & 1 & 2 & 1 \\
Valor Máximo & 2 & 2 & 2 & 192 \\
\hdashline Folhas mortas & 73 & 113 & 6 & 0 \\
\hline Número & 0 & 0 & 0 & 0 \\
Valor Mínimo & 0 & 1 & 1 & 4 \\
Moda & 2 & 4 & 2 & \\
Valor Máximo & & & & \\
\hline
\end{tabular}

\section{DISCUSSÃO E CONCLUSÕES}

O padrão de sobrevivência encontrado, representado por alta mortalidade nos estádios mais jovens, assemelha-se aos já descritos na literatura para E- ulis [18] e para outras espécies de palmœir- 2 2, 13, 21, 23]. A competição intraespecífi arente da alta densidade, tem sido apontada como um aos potenciais determinantes da mortalidade observada [4].

A mortalidade, como fenômeno que impede a alta ${ }^{4}$ densidade de palmito, relacionada às causas ambient ; e biológicas'veré' discutida por Reis (1995) [ enfatiza a predação e a ação de agentes__a mortalidade. Entretanto, para a população avaliada não foi possível detectar evidências da atuação de predadores e patógenos como agentes causadores FIGURA 1 - Freqüência o classesesn de crescimento
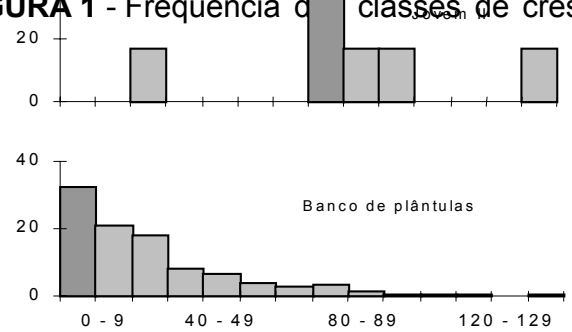

Classes de crescim ento em altura $(\mathrm{m} \mathrm{m})$ de mortalidade. Por outro lado, constatou-se que a mortalidade decorrente de danos físicos causados pela ${ }^{80} \mathrm{q}$ a de galhos ou folhas foi expressiva. Acreâfit a, também, que tal resultado foi subesti to porque muita ${ }_{\text {।a }}$ das indicações desse agente- danos podem ter se perdido ao longo do ano, a segunda coleta de dados. Futuros trabalhos poderiam avaliar melhor o papel dos danos

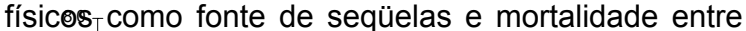
os indivíduos dos diferentes estádios dessa espécie e sua ortância na determinação da estrutura da popula

fator que poderia estar associado à sobrevivericra stia o tamanho das sementes. Observou-se que um dos adultos selecionados para o trabalho de acompanhamento fenológico produziu sementes visivelmente maiores que os outros.
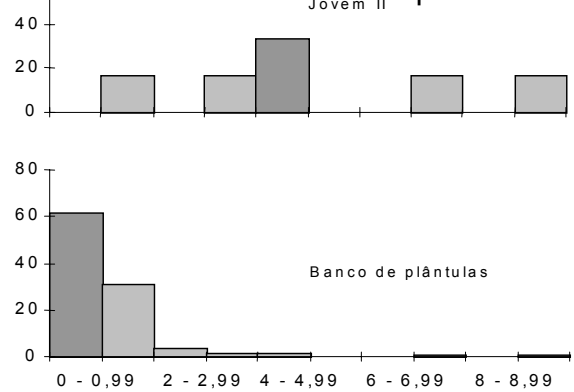

Classes de crescimento em diâm etro ( $m m$ 
em altura, para cada um dos estádios e para o banco de plântulas de Euterpes edulis, Paranaguá, PR, 1995/96 em diâmetro para cada um dos estádios e para o banco de plântulas de Euterpes edulis, Paranaguá, PR, 1995/96

FIGURA 2 - Freqüência das classes de crescimento
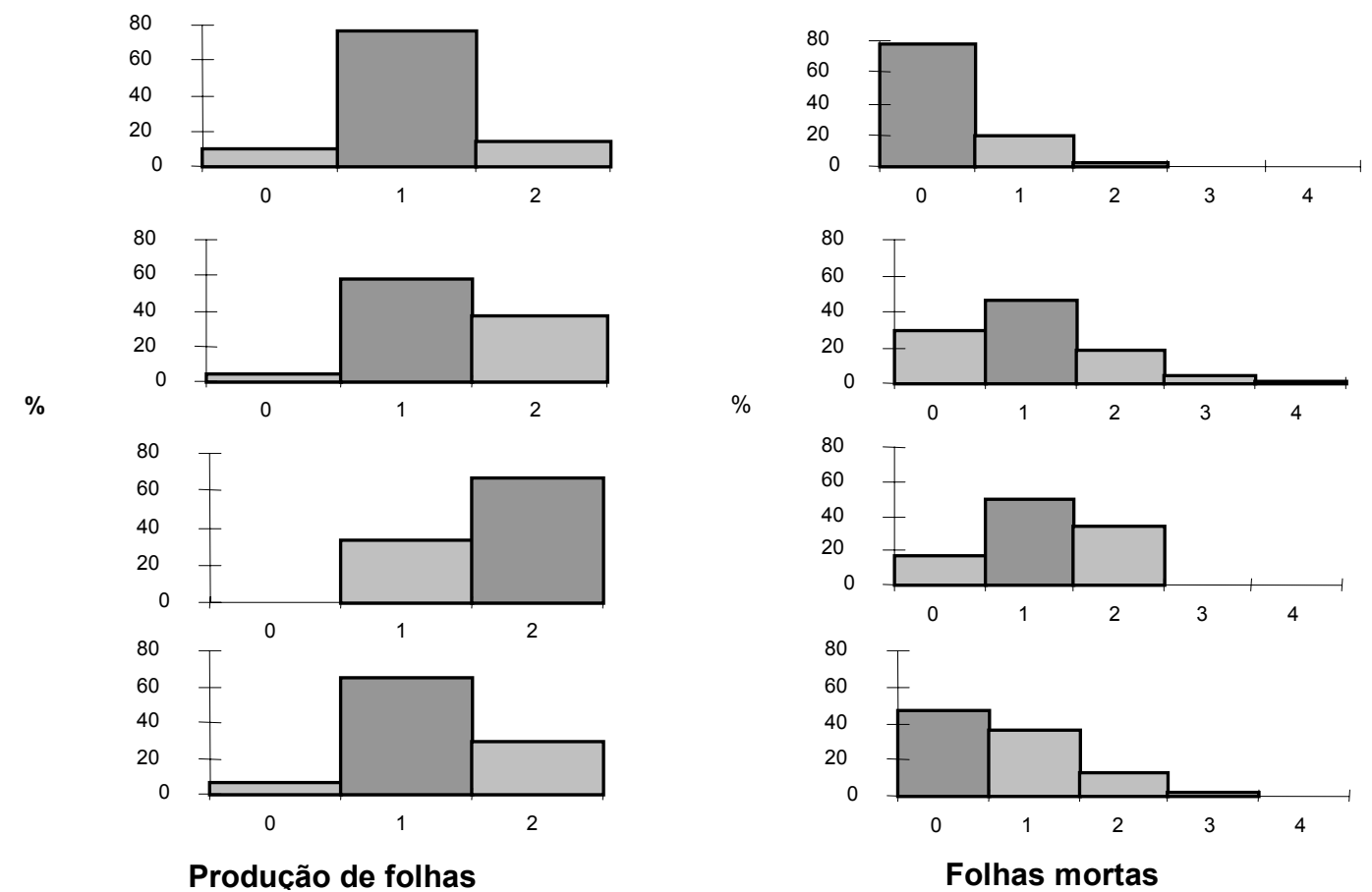

FIGURA 3 - Freqüência das classes de produção de folhas e número de folhas mortas em cd um dos estádios e para o banco de plântulas de Euterpes edulis, Paranaguá, PR, 1995/96

Tal característica está diretamente associada à melhor qualidade de germinação e vigor das sementes, como já evidenciado para E. edulis [9, 10]. Essa condição, portanto, poderia influenciar o crescimento e a sobrevivência das potenciais plântulas sob diferentes condições, como registrado para Virola surinamensis Warb., no Panamá [7].

A população estudada demonstrou aumento dos índices de crescimento do menor para o maior estádio de tamanho. Ash (1988) [1] e Ataroff (1992) [2] baseados em dados da produção de folhas encontraram resultado semelhante ao estudarem as palmeiras Balaka microcarpa Burret e Chamaedorea bartlingiana $\mathrm{H}$. Wendl., respectivamente. Para Ash (1988) [1], o número de folhas pode ser o parâmetro mais apropriado para predizer a velocidade da produção de folhas, na medida que aparentemente esta velocidade aumenta proporcionalmente ao número folhas expandidas.

Para Euterpe edulis, em média a produção anual de folhas foi maior que o de folhas mortas. Portanto, cada folha nova deve proporcionar ampliação da área fotossintética. Então, melhores condições de exposição à luz e maior área foliar devem ser os fatores responsáveis pelo aumento da produção de folhas do menor para o maior estádio de tamanho, por consequência também devem aumentar os valores de altura e diâmetro.
Detectou-se ampla variação do crescimento individual em cada um dos estádios analisados. Solbrig (1981) [19] demonstrou experimentalmente que as condições ambientais eram mais importantes que as diferenças genéticas entre os indivíduos na determinação do crescimento em Viola sororia Willd. Entretanto, isto não pode ser confirmado com base nos dados ambientais do local de estudo, que eram muito homogêneos, e em razão da variabilidade de crescimento observada.

Verificou-se que durante a coleta dos dados alguns dos indivíduos menores que $10 \mathrm{~cm}$ de altura apresentavam três folhas, fez-se necessário dar mais ênfase aos outros critérios citados na definição dos estádios, para o melhor enquadramento dos indivíduos no estádio de plântula ou jovem I. Optouse por enquadrar esses indivíduos no estádio jovem I, considerando que a presença de três folhas representaria melhor um indivíduo deste estádio que do estádio de plântula. O aprimoramento das definições dos diferentes estádios adotados poderia representar, com maior fidelidade, as principais fases de desenvolvimento da vida dos indivíduos de $E$. edulis. Com isso haveria maiores facilidades para o desenvolvimento de outras linhas de trabalho com esta espécie.

Conclusivamente pode se considerar que o registro de entrada de novos indivíduos na 
população e os valores de sobrevivência e transição obtidos para os diferentes estádios estudados são indicativos da franca regeneração natural do palmito no local de estudo. No entanto, o método de coleta dos dados de crescimento em altura e diâmetro precisam ser aperfeiçoados para que se possa ter valores mais representativos do real crescimento apresentado pelos indivíduos. Também precisam ser refinados os métodos de coleta das variáveis ambientais de locais visualmente homogêneos, como o estudado, para que se possa verificar a real influência dessas variáveis sobre o crescimento dos indivíduos. Uma das variáveis ambientais que necessitaria ser mais detalhada seria relativa aos níveis de subsuperfície, pois o horizonte $\mathrm{Bh}$ (impermeável) varia em profundidade, que por sua vez provoca variações na dinâmica do regime hídrico. Com isto, os indivíduos de diferentes pontos do local de estudo estão sujeitos a diferentes condições de suprimento de água $e$ conseqüentemente podem ter crescimento diferenciado em função dessa condição.

\section{AGRADECIMENTOS}

Os autores agradecem à Dra. Dalva M. Silva Matos e ao Dr. Ademir Reis pelas valiosas críticas e sugestões ao texto original, incluído na tese de mestrado do primeiro autor. À CAPES pela bolsa de mestrado cedida ao primeiro autor, que permitiu a realização da pesquisa. Aos dois consultores anônimos, pelas críticas e sugestões apresentadas.

\section{REFERÊNCIAS}

[1] ASH, J. Demography and production of Balaka microcarpa Burret (Arecaceae), a tropical understory palm in Fiji. Australian Journal of Botany, Melbourne, v.36, p. 67-80, 1988.

[2] ATAROFF, M.; SCHWARZKOPF, T. Leaf production, reproductive patterns, field germination and seedling survival in Chamaedorea bartlingiana, a dioecious understory palm. Oecologia, Berlin, v.92, p. 250-256, 1992.

[3] CLARK, D. B.; CLARK, D. A. The impact of physical damage on canopy tree regeneration in tropical rain forest. Journal of Ecology, Oxforf, v.79, p. 447-457, 1991.

[4] COOK, R. E. Patterns of juvenile mortality and recruitment in plants. In: SOLBRIG, O. T.; JAIN, S.; JOHNSON, G. B.; RAVEN, P. H. Topics in plant population biology. New York: Columbia University., 1979. p. 206-231.

[5] FLORIANO, E. P.; NODARI, R. O.; REIS, A.; REIS, M. S.; GUERRA, M. P. Manejo do palmiteiro: uma proposta. In: ENCONTRO NACIONAL DE PESQUISADORES EM PALMITO 1., 1987, Curitiba. Anais. Curitiba: EMBRAPA., 1987. p. 189-192.

[6] GENTRY, A. H. Patterns of neotropical plant species diversity. Evolutionary Biology, New York, v.15, p. 184, 1982.

[7] HOWE, H. F. Survival and growth of juvenile Virola surinamensis in Panama: effects of herbivory and canopy closure. Journal of Tropical Ecology, Cambridge, v.6, p. 259-280, 1990.

[8] IAPAR - Instituto Agronômico do Paraná. Cartas climáticas básicas do Estado do Paraná. Londrina: IAPAR, 1978. $41 \mathrm{p}$.

[9] LIN, S. S. Efeito de tamanho e maturidade sobre viabilidade, germinação e vigor do fruto de palmiteiro. Revista Brasileira de Sementes, Londrina, n.1, p. 5766, 1986.

[10]MARTINS, C. C.; NAKAGAWA, J.; BOVI, M. L. A. Dessication tolerance of four seeds lots of Euterpe edulis Mart. Seed Science and Technology, Zurich, v.28, n1, p. 1-13, 1999.

[11]NODARI, R. O.; REIS, A.; GUERRA, M. P.; REIS, M. S. Teste de procedência e progênie de palmiteiro (Euterpe edulis Mart.). In: ENCONTRO NACIONAL DE PESQUISADORES EM PALMITO 1., 1987, Curitiba. Anais. Curitiba: EMBRAPA, 1987. p. 183-188.

[12] OYAMA, K. Variation in growth and reproduction in the neotropical dioecious palm Chamaedorea tepejilote. Journal of Ecology, Oxforf, v.78, p. 648-663, 1990.

[13] PIÑERO, D.; MARTINEZ-RAMOS, M.; SARUKHÁN, J. A. Population model of Astrocaryum mexicanum and a sensitivity analysis of its finite rate of increase. Journal of Ecology, Oxford, v.72, p. 977-991, 1984.

[14] REIS, A. Dispersão de sementes de Euterpe edulis Martius - (Palmae) em uma Floresta Ombrófila Densa Montana da encosta atlântica em Blumenau, SC. Campinas, 1995. $154 \mathrm{f}$. Tese (Doutorado, Biologia Vegetal), Instituto de Biologia da Universidade de Campinas.

[15] REIS, A.; FANTINI, A. C.; REIS, M. S.; GUERRA, M. P.; NODARI, R. O. Manejo do palmiteiro Euterpe edulis em regime de rendimento sustentado. Florianópolis: Núcleo de Pesquisas em Florestas Tropicais, Universidade Federal de Santa Catarina, 1994. $59 \mathrm{p}$

[16] REIS, M. S.; NODARI, R. O.; GUERRA, M. P.; REIS, A. Desenvolvimento do palmiteiro: II. Avaliação preliminar a campo de mudas desenvolvidas sob diferentes níveis de sombreamento. In: ENCONTRO NACIONAL DE PESQUISADORES EM PALMITO 1., 1987, Curitiba. Anais. Curitiba: EMBRAPA, 1987. p. 193-194.

[17] SILVA, D. M. Estrutura de tamanho e padrão espacial de uma população de Euterpe edulis Mart. (Arecaceae) em mata mesófila semidecidua no Município de Campinas, SP. Campinas, 1992. $60 \mathrm{f}$. Dissertação (Mestrado em Ecologia), Universidade Estadual de Campinas.

[18]SILVA-MATOS, D. M. Population Ecology of Euterpe edulis Mart. (Palmae). England, 1995. $187 \mathrm{f}$. Tese (Doutorado), University of East Anglia Norwich Norfolk NR4TJ.

[19]SOLBRIG, O. T. Studies on the population biology of the Genus Viola: II. The effect of plant size on fitness in Viola sororia. Evolution, Laurence, v.35, n.6, p. 10801093, 1981.

[20] SPVS - Sociedade Protetora da Vida Selvagem. Plano integrado de conservação para a região de Guaraqueçaba, Paraná, Brasil. Curitiba: SPVS, 1992. $128 \mathrm{p}$.

[21] VANDERMEER, J. H. Notes on density dependence in Welfia georgii Wendl. ex Burret (palmae) a lowland rainforest species in Costa Rica. Brenesia, San Jose, v.10/11, p. 9-15. 1977.

[22] TONETTI, E. L. Estrutura da população, crescimento e dinâmica do banco de plântulas e fenologia reprodutiva de Euterpe edulis Mart. Arecaceae num trecho da Floresta Ombrofila Densa das Terras Baixas do Municipio de Paranaguá, PR. Curitiba, 1997. 63 f. Dissertação (Mestrado em Botânica) Setor de Ciências Biológicas, Universidade Federal do Paraná.

[23]VAN VALEN, L. Life, death and energy of a tree. Biotropica, New Orleans, v.7, n.4, p. 260-269, 1975.

[24]VELOSO, H. P.; RANGEL-FILHO, A. L. R.; LIMA, J. S. 
A. Classificação da vegetação brasileira, adaptada a um sistema universal. Rio de Janeiro: IBGE -
Departamento de Recursos Naturais e Estudos Ambientais, 1991. $123 \mathrm{p}$.

Recebido para publicação em 20 OUT 2000 [SA 032/2000] Aceito para publicação em 22 AGO 2001 